Research Article

\title{
$q$-Rung Orthopair Fuzzy Matroids with Application to Human Trafficking
}

\author{
Muhammad Asif $\mathbb{D D}^{1}$ Doha A. Kattan $\mathbb{D}^{2},{ }^{2}$ Dragan Pamučar $\mathbb{D}^{3},{ }^{3}$ and Ghous Ali $\mathbb{I D}^{4}$ \\ ${ }^{1}$ School of Mathematical Sciences, Xiamen University, Xiamen 361005, China \\ ${ }^{2}$ Department of Mathematics, Faculty of Sciences and Arts King Abdulaziz University, Rabigh, Saudi Arabia \\ ${ }^{3}$ Department of Logistics, Military Academy, University of Defence in Belgrade, Belgrade 11000, Serbia \\ ${ }^{4}$ Department of Mathematics, Division of Science \& Technology, University of Education, Lahore, Pakistan
}

Correspondence should be addressed to Doha A. Kattan; dakattan@kau.edu.sa

Received 11 July 2021; Revised 6 September 2021; Accepted 16 November 2021; Published 21 December 2021

Academic Editor: Chun Wei

Copyright (C 2021 Muhammad Asif et al. This is an open access article distributed under the Creative Commons Attribution License, which permits unrestricted use, distribution, and reproduction in any medium, provided the original work is properly cited.

\begin{abstract}
The theory of $q$-rung orthopair fuzzy sets ( $q$-ROFSs) is emerging for the provision of more comprehensive and useful information in comparison to their counterparts like intuitionistic and Pythagorean fuzzy sets, especially when responding to the models of vague data with membership and non-membership grades of elements. In this study, a significant generalized model $q$-ROFS is used to introduce the concept of $q$-rung orthopair fuzzy vector spaces ( $q$-ROFVSs) and illustrated by an example. We further elaborate the $q$-rung orthopair fuzzy linearly independent vectors. The study also involves the results regarding $q$-rung orthopair fuzzy basis and dimensions of $q$-ROFVSs. The main focus of this study is to define the concepts of $q$-rung orthopair fuzzy matroids ( $q$-ROFMs) and apply them to explore the characteristics of their basis, dimensions, and rank function. Ultimately, to show the significance of our proposed work, we combine these ideas and offer an application. We provide an algorithm to solve the numerical problems related to human flow between particular regions to ensure the increased government response action against frequently used path (heavy path) for the countries involved via directed $q$-rung orthopair fuzzy graph $(q$-ROFG). At last, a comparative study of the proposed work with the existing theory of Pythagorean fuzzy matroids is also presented.
\end{abstract}

\section{Introduction}

Graph theory and combinatorial geometry are known to have a lot of common grounds, particularly with regard to their basic concepts. Making use of these similarities, a host of research has been conducted for further exploration and development of these fields. Whitney [1] was the one to initiate the fundamental concept of matroids. By doing so, he laid the foundation of an extremely vast field of matroid theory that connected several basic tools like linear algebra, graph, and combinatorial theory. This matroid theory has been widely applied by researchers in different scientific areas.

Zadeh [2], in 1965, for the first time introduced fuzzy logics and defined fuzzy sets (FSs). These sets were known for real-life data, uncertainties, and vague information. Soon after its introduction, fuzzy set theory became popular among researchers and came up as a new field. Later, Attanssov [3] expanded the concept of FSs and introduced the intuitionistic fuzzy sets (IFSs) with the help of membership and non-membership values of elements, the sum of which was not being more than 1 . These IFSs are effectively applied in theoretical as well as practical problems such as optimization, decision making, and graphical ones in numerous fields. The idea of these sets was further extended by Feng et al. [4] to give intuitionistic fuzzy soft sets (IFSSs). They also presented several new operations to generalize the concept of intuitionistic fuzzy soft sets (IFSSs). While solving some decision-making problems, it was observed that the sum of both membership and non-membership values of elements exceeded one; however, some of their squares remained less than one. To overcome such issues, 
Yager $[5,6]$ put forward the idea of IFSs with the introduction of new Pythagorean fuzzy sets. Some useful notions and results for FSs, IFSs, PFSs, and other types of fuzzy sets have been presented in the literature [7-14]. It seems difficult to solve the problems when the sum of the square of the membership and non-membership values of elements exceeds 1 . We are unable to handle such kind of information by means of PFSs. Yager [15] introduced $q$-ROFSs in which the sum of the $q$ th power of membership and nonmembership values of elements is bounded by one. After that, $q$-ROFSs are frequently used in decision making as $q$-ROFSs widened the range of acceptable pairs rather than IFSs and PFSs with the parameter " $q$ " adjustment. Recently, Garg used $q$-ROFSs to introduce a novel concept of connection number-based $q$-rung orthopair fuzzy set (CN-q-ROFS), defined some operation laws, and proposed a method to handle multiattribute group decision-making (MAGDM) problems [16]. Subsequently, in [17], he introduced the idea of $q$-connection numbers for interval-valued $q$-rung orthopair fuzzy set and used it to develop a method for solving multiattribute group decision-making (MAGDM) problems. At present, several studies paid close attention to the information regarding $q$-ROFSs and provided different novel methods [18-21].

The graphical representation of objects has been a subject of great interest for scientists. Recently, a number of studies have involved both fuzzy and graph theories to deal with the optimization related problems in the presence of vague data. The idea of fuzzy graphs came from Kaufmann [22], while some basic concepts related to fuzzy graphs, such as cycles and paths, were characterized by Rosenfield et al. [23]. Akram and Naz [24] further used these concepts and proposed a new work to find the energy of PFGs with their applications. Their work was mainly focused on operations of fuzzy graphs (FGs), IFGs, PFGs, and their different types. They also provided $q$-ROF competition graphs and studied their applications. Sitara et al. [25] introduced the notion of $q$-rung picture fuzzy graph structures and provided an algorithm to describe their proposed model. The refining of the idea of hypergraphs given by Kaufmann was carried out by Lee-Kwang and Lee. In addition, different researchers investigated numerous features of FGs and fuzzy hypergraphs based on different FSs [26-31]. In 1988, the concept of matroids in terms of FSs was linked and defined as G-V fuzzy matroids by Goetschel and Voxman [32]. Later on, bases and circuits of the fuzzy matroids were also defined by them [33-36]. As time progressed, different FSs were used to define different fuzzy matroids, and their properties were also discussed by different researchers [37-42]. Recently, we proposed the idea of Pythagorean fuzzy matroids (PFMs) and described their application to decision making [43]. A lot of work based on FSs, IFSs, and PFSs regarding matroid theory has been discussed in the literature, but matroids based on $q$-ROFSs are still unattended. The existing models, namely, IFMs and PFMs, are insufficient to deal with different decision-making problems which contain membership and nonmembership values of elements whose sum of their squares is greater than 1. This drawback of existing structures motivates us to present this work.

The motivations of our work are as follows:

(1) The $q$-ROFS is a generalized form of some existing models, including IFSs and PFSs. On setting $q=1$ and $q=2$, we get IFSs and PFSs, respectively, as special cases of $q$-ROFSs.

(2) The existing IFMs and PFMs fail to deal with the information involving membership and nonmembership values whose sum of their squares is not less than 1 .

(3) Due to the more flexible approach of $q$-ROFSs, the developed $q$-ROFMs can solve many decisionmaking problems and overcome deficiencies of existing models such as IFMs and PFMs.

The main contributions of this work are as follows:

(1) Our work illustrates $q$-ROFVSs with an illustrative example.

(2) Most importantly, the notion of $q$-ROFMs is defined and characterized with its basis and dimension.

(3) This study also provides various results regarding q-ROFMs.

(4) Ultimately, an algorithm is developed to find an optimal solution along with a particular application.

(5) To check the validity of our proposed work, a comparative analysis with an existing model is also given.

In this work, we present the idea of $q$-ROFVSs with a numerical example and discuss their bases and dimensions. We also discuss the $q$-rung orthopair fuzzy linearly independent vectors. We further combined the $q$-ROFSs with the fuzzy matroids and named them as $q$-ROFMs. We investigate the concepts of circuits, basis, and rank for $q$-ROFMs. Note that for $q=1$ and $q=2$, our proposed $q$-ROFMs are reduced to IFMs and PFMs, respectively. We also proposed an application of our work regarding human trafficking between different regions which supports them to find a heavy path used by the traffickers so that they can increase their government response action against this path by using a directed graph having $q$-rung orthopair fuzzy information. In the end, we give concluding remarks with some of the future directions.

The contents of this article are summarized as follows. In Section 2, we recall some fundamental definitions including crisp matroids with rank function, $q$-ROFSs with their score functions, and some basic operations defined on $q$-ROFSs. In Section 3, we first propose $q$-ROFVSs and then $q$-ROFMs. We also discuss some of their basic properties in this section. In Section 4, we explore an application and develop an algorithm to illustrate the importance of our work. In Section 5, we provide the numerical comparison of our developed algorithm with the existing PFM approach [43]. In Section 6, we provide some conclusive remarks with future directions. 


\section{Preliminaries}

Our interest in this section is to discuss the theory of matroids and valuable concepts related to matroid theory to understand the proposed work better. Although matroids are defined differently by using various sets, here we write a simple definition of crisp matroids.

Definition 1 (see [1]). Let $A \neq \phi$ be a set of finite elements and $P(A)$ denote the power set of $A$. For $\mathscr{I} \subset P(A)$, a nonempty family of subsets, the pair $\mathscr{M}=(A, \mathscr{J})$ is called a matroid (or crisp matroid) if it satisfies the following:

(1) $\phi \in \mathscr{I}$.

(2) If $I_{2} \subset I_{1}$ with $I_{1} \in \mathscr{I}$, then $I_{2} \in \mathscr{I}$.

(3) If $I_{1}, I_{2} \in \mathscr{I}$ with $\left|I_{2}\right|<\left|I_{1}\right|$, then another subset $I_{3} \in \mathscr{I}$ exists such that $I_{2} \subset I_{3} \subseteq I_{1} \cup I_{2}$, where $|I|$ shows the number of elements of $I$.

The element $I \in \mathscr{I}$ is called independent set in $\mathscr{M}$. Also, $I$ is known as maximal independent in $\mathscr{M}$ if we do not have such $I^{\prime} \in \mathscr{I}$ that contains $I$.

Definition 2 (see [1]). Suppose that $\mathscr{M}$ is a matroid and $I \in \mathscr{F}$. If $I$ is maximal independent in $\mathscr{M}$, then $I$ is called base of $\mathscr{M}$ and $B(\mathscr{M})$ represents the family of all bases.

Definition 3. Let $J \in P(A)$ be a subset with $J \notin I$; then, $J$ is called dependent subset. A circuit of $\mathscr{M}$ is the subset $J \in P(A) \backslash \mathscr{I}$ where $J$ is inclusion-wise minimal dependent subset.

Definition 4 (see [1]). Let $\mathscr{M}=(A, \mathscr{F})$ be a matroid. Consider a mapping $\mathscr{R}_{A}: P(A) \longrightarrow\{0,1,2, \ldots,|A|\}$ defined as

$$
\mathscr{R}_{A}(A)=\max \left\{\left|I^{\prime}\right|: I^{\prime} \subset A, I \in \mathscr{I}\right\} .
$$

Then, $\mathscr{R}_{A}$ is called rank function of $\mathscr{M}$.

Definition 5 (see [2]). Consider a non-empty set $X$. The fuzzy set $F$ is defined as

$$
F=\left\{\left(x, \sigma_{F}(x)\right) \mid x \in X\right\} .
$$

The mapping $\sigma_{F}: X \longrightarrow[0,1]$ assigns the membership value of $x \in X$ to $F$ and $\mathscr{F} \mathcal{S}(X)$ represents the family of all fuzzy sets on $X$.

Definition 6 (see [32]). Let $X$ be a non-empty finite universe of discourse and $\mathscr{F} \subseteq \mathscr{F} \mathcal{S}(X)$. For any fuzzy sets $F_{1}, F_{2}, F_{3} \in \mathscr{F} \mathcal{S}(X)$, the collection $\mathscr{F}$ satisfies the following:

(1) $\phi \in \mathscr{F}$.

(2) If $F_{1} \subset F_{2}$ and $F_{2} \in \mathscr{F}$, then $F_{1} \in \mathscr{F}$, where $F_{1} \subset$ $F_{2}$ means $F_{1}(x)<F_{2}(x)$, for all $x \in X$.

(3) Let $F_{1}, F_{2} \in \mathscr{F}$ and $\left|\sup p\left(F_{1}\right)\right|<\left|\sup p\left(F_{2}\right)\right|$ where $\sup p(F)=\{x \in X \mid F(x)>0\}$ and $|F|=\sum_{x \in X}(F(x))$; then, another $F_{3} \in \mathscr{F}$ exists satisfying

(a) $F_{1} \subset F_{3} \subseteq F_{1} \cup F_{2}$, for any $x \in X$, and union is defined as $\left(F_{1} \cup F_{2}\right)(x)=\max \left\{F_{1}(x), F_{2}(x)\right\}$.

(b) $m\left(F_{3}\right) \geq \min \left\{m\left(F_{1}\right), m\left(F_{2}\right)\right\}$, and $m\left(F_{i}\right)=$ $\min \left\{F_{i}(x): x \in \sup p\left(F_{i}\right)\right\}$ is defined for any $x \in X$.

The pair $\mathscr{F} \mathscr{M}(X)=(X, \mathscr{F})$ is called a fuzzy matroid and $\mathscr{F}$ is the subfamily of all independent FSs of the matroid $\mathscr{F} \mathscr{M}(X)$.

Definition 7 (see [15]). Let $X$ be a non-empty fixed set with finite elements. Then, the set $\zeta=\left(\zeta^{+}, \zeta^{-}\right)$defined on a fixed set $X$ is called $q$-ROFS if it satisfies

(1) $q \geq 1$.

(2) $\zeta^{+}(x) \in[0,1]$ and $\zeta^{-}(x) \in[0,1]$ with the property $\left(\zeta^{+}(x)\right)^{q}+\left(\zeta^{-}(x)\right)^{q} \leq 1$, for any $x \in X$.

It can be seen easily that for $q=1$ and $q=2$, these fuzzy sets are reduced to IFS and PFS, respectively. The $q$-rung orthopair degree of hesitance for $x \in X$ is given as

$$
\varrho_{\zeta}(x)=\sqrt[q]{1-\left(\zeta^{+}\right)^{q}-\left(\zeta^{-}\right)^{q}}
$$

Definition 8 (see [15]). Let $\left(\zeta^{+}, \zeta^{-}\right)$be a $q$-ROFS and for $x \in X,\left(\zeta^{+}(x), \zeta^{-}(x)\right)$ be a $q$-rung orthopair fuzzy number $\left(q\right.$-ROFN). Then, a score function $\delta_{\tilde{q}}$ of $\left(\zeta^{+}(x), \zeta^{-}(x)\right)$ is defined as

$$
\mathcal{S}_{\tilde{q}}\left(\zeta^{+}(x), \zeta^{-}(x)\right)=\frac{1}{2}\left(1+\left(\zeta^{+}(x)\right)^{q}-\left(\zeta^{-}(x)\right)^{q}\right), \quad 0 \leq \mathcal{S}_{\tilde{q}}\left(\zeta^{+}(x), \zeta^{-}(x)\right) \leq 1 .
$$

Definition 9 (see [15]). Let $\zeta=\left(\zeta^{+}, \zeta^{-}\right)$be a $q$-ROFS and for $x \in X, \quad\left(\zeta^{+}(x), \zeta^{-}(x)\right)$ be a $q$-ROFN. Then, for any $x \in X$, an accuracy function $\mathscr{A}_{\tilde{q}}$ for $q$-ROFN is defined as 


$$
\mathscr{A}_{\tilde{q}}\left(\zeta^{+}(x), \zeta^{-}(x)\right)=1-\varrho_{\zeta}(x) .
$$

Definition 10 (see [41]). Let $\zeta_{1}$ and $\zeta_{2}$ be two $q$-ROFSs. Then, for any $\quad x \in X, \quad \zeta_{1} \cup \zeta_{2}=\left(\zeta_{1}^{+}, \zeta_{1}^{-}\right) \vee\left(\zeta_{2}^{+}, \zeta_{2}^{-}\right)$and $\zeta_{1} \cap \zeta_{2}=\left(\zeta_{1}^{+}, \zeta_{1}^{-}\right) \wedge\left(\zeta_{2}^{+}, \zeta_{2}^{-}\right)$are called union and intersection, respectively, defined as

$$
\begin{aligned}
&\left(\zeta_{1} \cup \zeta_{2}\right)(x)= \begin{cases}\left(\zeta_{1}^{+}, \zeta_{1}^{-}\right)(x), & \text { if } \mathcal{S}_{\tilde{q}}\left(\zeta_{1}^{+}, \zeta_{1}^{-}\right)(x)>\mathcal{S}_{\tilde{q}}\left(\zeta_{2}^{+}, \zeta_{2}^{-}\right)(x), \\
\left(\zeta_{1}^{+}, \zeta_{1}^{-}\right)(x), & \text { if } \mathcal{S}_{\tilde{q}}\left(\zeta_{1}^{+}, \zeta_{1}^{-}\right)(x)=\mathcal{S}_{\tilde{q}}\left(\zeta_{2}^{+}, \zeta_{2}^{-}\right)(x) \text { and } \mathscr{A}_{\tilde{q}}\left(\zeta_{1}^{+}, \zeta_{1}^{-}\right)(x) \geq \mathscr{A}_{\tilde{q}}\left(\zeta_{2}^{+}, \zeta_{2}^{-}\right)(x), \\
\left(\zeta_{2}^{+}, \zeta_{2}^{-}\right)(x), & \text { if } \mathcal{S}_{\tilde{q}}\left(\zeta_{1}^{+}, \zeta_{1}^{-}\right)(x)=\mathcal{S}_{\tilde{q}}\left(\zeta_{2}^{+}, \zeta_{2}^{-}\right)(x) \text { and } \mathscr{A}_{\tilde{q}}\left(\zeta_{1}^{+}, \zeta_{1}^{-}\right)(x)<\mathscr{A}_{\tilde{q}}\left(\zeta_{2}^{+}, \zeta_{2}^{-}\right)(x),\end{cases} \\
&\left(\zeta_{1} \cap \zeta_{2}\right)(x)= \begin{cases}\left(\zeta_{2}^{+}, \zeta_{2}^{-}\right)(x), & \text { if } \mathcal{S}_{\tilde{q}}\left(\zeta_{1}^{+}, \zeta_{1}^{-}\right)(x)>\mathcal{S}_{\tilde{q}}\left(\zeta_{2}^{+}, \zeta_{2}^{-}\right)(x), \\
\left(\zeta_{2}^{+}, \zeta_{2}^{-}\right)(x), & \text { if } \mathcal{S}_{\tilde{q}}\left(\zeta_{1}^{+}, \zeta_{1}^{-}\right)(x)=\mathcal{S}_{\tilde{q}}\left(\zeta_{2}^{+}, \zeta_{2}^{-}\right)(x) \text { and } \mathscr{A}_{\tilde{q}}\left(\zeta_{1}^{+}, \zeta_{1}^{-}\right)(x) \geq \mathscr{A}_{\tilde{q}}\left(\zeta_{2}^{+}, \zeta_{2}^{-}\right)(x), \\
\left(\zeta_{1}^{+}, \zeta_{1}^{-}\right)(x), & \text { if } \mathcal{S}_{\tilde{q}}\left(\zeta_{1}^{+}, \zeta_{1}^{-}\right)(x)=\mathcal{S}_{\tilde{q}}\left(\zeta_{2}^{+}, \zeta_{2}^{-}\right)(x) \text { and } \mathscr{A}_{\tilde{q}}\left(\zeta_{1}^{+}, \zeta_{1}^{-}\right)(x)<\mathscr{A}_{\tilde{q}}\left(\zeta_{2}^{+}, \zeta_{2}^{-}\right)(x)\end{cases}
\end{aligned}
$$

Here, we denote the family of all $q$-ROFSs on $X$ by $\mathbb{Q}(X)$. Let $\zeta=\left\langle x, \zeta^{+}(x), \zeta^{-}(x) \mid x \in X\right\rangle$ be a $q$-ROFS; then, some notions are defined as follows.

(1) $|\zeta|=\sum_{x \in X}\left(\zeta^{+}(x), \zeta^{-}(x)\right)$.

(2) $\operatorname{Sup} p\left(\zeta^{+}, \zeta^{-}\right)=\left\{x \in X: \mathcal{S}_{\tilde{q}}\left(\zeta^{+}(x), \zeta^{-}(x)\right)>0\right\}$.

(3) $\mathscr{Q}_{q}\left(\zeta^{+}, \zeta^{-}\right)=\left\{x \in X: \mathcal{S}_{\tilde{q}}\left(\zeta^{+}(x), \zeta^{-}(x)\right) \geq q\right\}, \quad$ for $0 \leq q \leq 1$.

(4) $\mathscr{R}^{+}\left(\zeta^{+}, \zeta^{-}\right)=\left\{\mathcal{S}_{\tilde{q}}\left(\zeta^{+}(x), \zeta^{-}(x)\right) \mid \delta_{\tilde{q}} \quad\left(\zeta^{+}(x), \zeta^{-}\right.\right.$ $(x))>0\}$. A $q$-ROFS $\left(\zeta^{+}, \zeta^{-}\right)$is called elementary if $\mathscr{R}^{+}\left(\zeta^{+}, \zeta^{-}\right)=1$.

(5) $h(\zeta)=\max _{x \in X}\left(\zeta^{+}(x), \zeta^{-}(x)\right)$ is called height of $\zeta$ and the set $\zeta$ is called normal $q$-ROFS for the height $h(\zeta)=(1,0)$.

Note that we denote the smallest and the largest $q$-rung orthopair fuzzy elements $\mathbf{0}=(0,1)$ and $\mathbf{1}=(1,0)$, respectively.

\section{3. $q$-Rung Orthopair Fuzzy Vector Spaces}

This section illustrates the concept of $q$-ROFVSs with their basis and dimension and presents $q$-rung orthopair fuzzy linearly dependent and independent vectors. Here, we also present matroids based on $q$-ROFSs and discuss their properties regarding circuits, basis, and their rank function. Katsaras and Liu [44] introduced the hybrid concept of fuzzy vector spaces and discussed their characteristics. Later, many researchers applied different fuzzy sets to the elementary concepts of vector spaces. Here, we use $q$-ROFSs to generalize the Pythagorean fuzzy vector spaces [43] and define $q$-ROFVSs.

Definition 11. Let $X$ be a non-empty finite vector space over the field $\mathbb{F}$. The $q$-ROFS $\zeta=\left(\zeta^{+}(x), \zeta^{-}(x)\right)$, for $x \in X$, is called $q$-ROFVS over $X$, if for scalars $a, b \in \mathbb{F}$ and for any $x, y \in X$, we have

$$
\begin{aligned}
\zeta^{+}(a x+b y) & \geq \min \left\{\zeta^{+}(x), \zeta^{+}(y)\right\}, \\
\zeta^{-}(a x+b y) & \leq \max \left\{\zeta^{-}(x), \zeta^{+}(y)\right\},
\end{aligned}
$$

where $0 \leq\left(\zeta^{+}(x)\right)^{q}+\left(\zeta^{-}(x)\right)^{q} \leq 1$ holds for defined mappings $\zeta^{+}: X \longrightarrow[0,1]$ and $\zeta^{-}: X \longrightarrow[0,1]$.

Here, the set of all $q$-ROFVSs over $X$ is denoted by the pair $\widetilde{X}=(X, \zeta)$.

The following proposition illustrates that membership and non-membership functions assign unchanged values under scalar multiplication in $q$-ROFVSs.

Proposition 1. Let $\widetilde{X}=(X, \zeta)$ be a $q$-ROFVS. The following two properties hold for each $\mathbf{x}, \mathbf{y} \in X$ :

(1) $\zeta^{+}(0,0)=\max _{\mathbf{x} \in X} \zeta^{+}(\mathbf{x})$ and $\zeta^{-}(0,0)=\min _{\mathbf{x} \in X} \zeta^{-}$ $(\mathbf{x})$.

(2) For any non-zero scalar $k \in \mathbb{F}$, $\zeta^{+}(k \mathbf{x})=\zeta^{+}(\mathbf{x})$ and $\zeta^{-}(k \mathbf{x})=\zeta^{-}(\mathbf{x})$

Proof. The proof of properties (15) and (18) is very straightforward (see Definition 11).

Proposition 2. Let $x, y \in X$ with $\zeta^{+}(\mathbf{x}) \neq \zeta^{+}(\mathbf{y})$ and $\zeta^{-}(\mathbf{x}) \neq \zeta^{-}(\mathbf{y})$; then, we have

$$
\begin{array}{r}
\zeta^{+}(\mathbf{x}+\mathbf{y}) \geq \min \left\{\zeta^{+}(\mathbf{x}), \zeta^{+}(\mathbf{y})\right\} \\
\zeta^{-}(\mathbf{x}+\mathbf{y}) \leq \max \left\{\zeta^{-}(\mathbf{x}), \zeta^{-}(\mathbf{y})\right\} .
\end{array}
$$

Proof. To prove, from Definition 11, let $a=b=1$ and hence $\zeta^{+}(\mathbf{x}+\mathbf{y}) \geq \min \left\{\zeta^{-}(\mathbf{x}), \zeta^{+}(\mathbf{y})\right\}$ and $\zeta^{-}(\mathbf{x}+\mathbf{y}) \leq \max \left\{\zeta^{-}(\mathbf{x})\right.$, $\left.\zeta^{-}(\mathbf{y})\right\}$.

Definition 12. Let $X$ be a non-empty finite universe and $\widetilde{X}$ be a $q$-ROFVS over $\mathbb{F}$. Then, the set of vectors $\left\{x_{1}, x_{2}, x_{3}, \ldots, x_{r}\right\} \subset X$ is called a $q$-rung orthopair fuzzy $(q$-ROF) linearly independent in $\widetilde{X}$ if

(1) $\left\{x_{1}, x_{2}, \ldots, x_{r}\right\}$ is linearly independent.

(2) For any $\left\{a_{1}, a_{2}, \ldots, a_{r}\right\} \subset \mathbb{F}$, we have 


$$
\begin{aligned}
\zeta^{+}\left(\sum_{j=1}^{r} a_{j} x_{j}\right) & =\min _{j=1}^{r} \zeta^{+}\left(a_{j} x_{j}\right), \\
\zeta^{-}\left(\sum_{j=1}^{r} a_{j} x_{j}\right) & =\max _{j=1}^{r} \zeta^{-}\left(a_{j} x_{j}\right) .
\end{aligned}
$$

Proposition 3. Let $\widetilde{X}=(X, \zeta)$ be a q-ROFVS over $\mathbb{F}$. Consider any set of vectors with non-zero elements $\left\{\alpha_{i}\right\}_{i=1}^{r} \in X \backslash\{0\}$ such that for $i \neq j, \zeta\left(\alpha_{i}\right) \neq \zeta\left(\alpha_{j}\right)$. Then, the set $\left\{\alpha_{i}\right\}_{i=1}^{r}$ is linearly and $q$-ROF linearly independent.

Proof. By using the induction on $r$, the statement is true for $r=1$. We suppose that the statement is true for $r$. So, $\left\{\alpha_{i}\right\}_{i=1}^{r}$ is $q$-ROF linearly independent. Let $\left\{\alpha_{i}\right\}_{i=1}^{r+1} \in X,\{0\}$ such that for $i \neq j, \quad\left(\zeta^{+}\left(\alpha_{i}\right), \zeta^{-}\left(\alpha_{i}\right)\right) \neq\left(\zeta^{+}\left(\alpha_{j}\right), \zeta^{-}\left(\alpha_{j}\right)\right)$ and suppose that the set $\left\{\alpha_{i}\right\}_{i=1}^{r+1}$ is not linearly independent. Thus, for $\phi \neq v \subset\{1,2, \ldots, r\}$, we have $\alpha_{r+1}=\sum_{i \in v}\left(a_{i} \alpha_{i}\right)$ where $a_{i} \neq 0$ for all $i \in v$. Then,

$$
\begin{aligned}
& \zeta^{+}\left(\alpha_{r+1}\right)=\min _{i \in v}\left(\zeta^{+}\left(a_{i} \alpha_{i}\right)\right)=\min _{i \in v}\left(\zeta^{+}\left(\alpha_{i}\right)\right) \in\left\{\zeta^{+}\left(\alpha_{i}\right)\right\}_{i=1}^{r}, \\
& \zeta^{-}\left(\alpha_{r+1}\right)=\max _{i \in v}\left(\zeta^{-}\left(a_{i} \alpha_{i}\right)\right)=\max _{i \in v}\left(\zeta^{-}\left(\alpha_{i}\right)\right) \in\left\{\zeta^{-}\left(\alpha_{i}\right)\right\}_{i=1}^{r},
\end{aligned}
$$

which gives that $\left(\zeta^{+}\left(\alpha_{r+1}\right), \zeta^{-}\left(\alpha_{r+1}\right)\right) \in\left\{\left(\zeta^{+}\left(\alpha_{i}\right), \zeta^{-}\left(\alpha_{i}\right)\right)\right\}_{i=1}^{r}$ and contradicts that $\left\{\alpha_{i}\right\}_{i=1}^{r+1}$ has distinct values and hence is linearly independent. Propositions 1 and 2 show that $\left\{\alpha_{i}\right\}_{i=1}^{r}$ is $q$-ROF linearly independent.

Definition 13. Let $\widetilde{X}=(X, \zeta)$ be a $q$-ROFVS and $\mathscr{B}=\left\{\beta_{j}\right\}_{j=1}^{r}$, where each $\beta_{j} \in X$. Then, the set $\mathscr{B}$ is called $q$-ROF basis in $\tilde{X}$, if it satisfies

(1) The set $\mathscr{B}$ is basis in $X$.

(2) For scalars $\left\{a_{1}, a_{2}, \ldots, a_{r}\right\} \subset \mathbb{F}$, we have

$$
\begin{aligned}
& \zeta^{+}\left(\sum_{j=1}^{r} a_{j} \beta_{j}\right)=\min _{j=1}^{r} \zeta^{+}\left(a_{j} \beta_{j}\right), \\
& \zeta^{-}\left(\sum_{j=1}^{r} a_{j} \beta_{j}\right)=\max _{j=1}^{r} \zeta^{-}\left(a_{j} \beta_{j}\right) .
\end{aligned}
$$

Definition 14. Let $X \neq \phi$ and $\tilde{X}$ be a $q$-ROFVS having basis $\mathscr{B}$. Then, the dimension of $q$-ROFVS is given by

$$
\operatorname{dim}_{q}(\tilde{X})=\sup _{\mathscr{B}}\left(\sum_{x \in \mathscr{B}}\left(\zeta^{+}(x), \zeta^{-}(x)\right)\right) .
$$

It is easy to see that $\operatorname{dim}_{q}$ is a function from the class of all $q$-ROFVSs to $[0, \infty) \cup\{\infty\}$. A $q$-ROFVS is said to be finite dimensional if and only if $\operatorname{dim}_{q}(\tilde{X})=n<\infty$.

Proposition 4. Let $\tilde{X}=(X, \zeta)$ be a $q-R O F V S$ over $\mathbb{F}$. For any $x, y \in X$, if $\zeta^{+}(x)>\zeta^{+}(y)$ and $\zeta^{-}(x)<\zeta^{-}(y)$, we have

$$
\begin{aligned}
& \zeta^{+}(x+y)=\zeta^{+}(y), \\
& \zeta^{-}(x+y)=\zeta^{-}(y) .
\end{aligned}
$$

Proof. We use Proposition 2:

$$
\zeta^{+}(x+y) \geq \min \left\{\zeta^{+}(x), \zeta^{+}(y)\right\} .
$$

Since $\zeta^{+}(x)>\zeta^{+}(y)$, then

$$
\zeta^{+}(x+y) \geq \zeta^{+}(y)
$$

Now we write

$$
\zeta^{+}((x+y)-x)=\zeta^{+}(y)
$$

which implies the result

$$
\zeta^{+}(y) \geq \min \left\{\zeta^{+}(x+y), \zeta^{+}(x)\right\}
$$

Since $\zeta^{+}(x)>\zeta^{+}(y)$, then

$$
\zeta^{+}(y) \geq \zeta^{+}(x+y)
$$

which proves that $\zeta^{+}(x+y)=\zeta^{+}(y)$. Similarly, we use Proposition 2:

$$
\zeta^{-}(x+y) \leq \max \left\{\zeta^{-}(x), \zeta^{-}(y)\right\} .
$$

Since $\zeta^{-}(x)<\zeta^{-}(y)$, then

$$
\zeta^{-}(x+y) \leq \zeta^{-}(y) .
$$

Now we write

$$
\zeta^{-}((x+y)-x)=\zeta^{-}(y)
$$

which implies the result

$$
\zeta^{-}(y) \leq \max \left\{\zeta^{-}(x+y), \zeta^{-}(x)\right\} .
$$

Since $\zeta^{-}(x)<\zeta^{-}(y)$, then

$$
\zeta^{-}(y) \leq \zeta^{-}(x+y)
$$

which proves that $\zeta^{-}(x+y)=\zeta^{-}(y)$.

The following example illustrates Definition 11 clearly.

Example 1. Let $X=\mathbb{R}^{3}$ and $\zeta=\left(\zeta^{+}, \zeta^{-}\right)$be a 5-ROFS defined on $X$. For any $\alpha=(x, y, z) \in \mathbb{R}^{3}$, the mappings $\zeta^{+}: \mathbb{R}^{3} \longrightarrow[0,1]$ and $\zeta^{-}: \mathbb{R}^{3} \longrightarrow[0,1]$ are defined by 
$\zeta^{+}(\alpha)= \begin{cases}\frac{1}{2}, & \text { if }(0,0,0), \\ \frac{1}{4}, & \text { if }(\mathbb{R} \backslash\{0\}, 0,0),(0, \mathbb{R} \backslash\{0\}, 0) \text { or }(0,0, \mathbb{R} \backslash\{0\}), \\ \frac{1}{2}, & \text { otherwise, }\end{cases}$
$\zeta^{-}(\alpha)=\left\{\begin{array}{lr}0, & \text { if }(0,0,0), \\ \frac{1}{4}, & \text { if }(\mathbb{R} \backslash\{0\}, 0,0),(0, \mathbb{R} \backslash\{0\}, 0) \text { or }(0,0, \mathbb{R} \backslash\{0\}), \\ \text { otherwise, } & \end{array}\right.$

respectively. It can be easily seen that, for $\alpha_{1}=\alpha_{2}=(0,0,0)$, the case is trivial.

For the second case, consider two vectors $\alpha_{1}$ and $\alpha_{2}$ from $X$ with one non-zero component, i.e., $\alpha_{1}(x, 0,0)$ and $\alpha_{2}(0, y, 0)$; then, we have $\min \left\{\zeta^{+}\left(\alpha_{1}\right), \zeta^{+}\left(\alpha_{2}\right)\right\}=(1 / 4)$ and $\max \left\{\zeta^{-}\left(\alpha_{1}\right), \zeta^{-}\left(\alpha_{2}\right)\right\}=(1 / 4)$. For any $a, b \in \mathbb{R}$, we have

$$
\begin{aligned}
& \zeta^{+}\left(a \alpha_{1}+b \alpha_{2}\right)=\zeta^{+}(a x, b y, 0)= \begin{cases}\frac{1}{2}, & \text { if } a=b=0 \text { or } a \neq 0, b \neq 0, \\
\frac{1}{4}, & \text { if } a=0 \text { or } b=0,\end{cases} \\
& \zeta^{-}\left(a \alpha_{1}+b \alpha_{2}\right)=\zeta^{-}(a x, b y, 0)= \begin{cases}0, & \text { if } a=b=0 \text { or } a \neq 0, b \neq 0, \\
\frac{1}{4}, & \text { if } a=0 \text { or } b=0 .\end{cases}
\end{aligned}
$$

Clearly, it satisfies all the conditions of Definition 11.

Now, consider $\alpha_{1}$ and $\alpha_{2} \in X$ with one zero component and two non-zero components, i.e., $\alpha_{1}\left(x_{1}, y_{1}, 0\right)$ and $\alpha_{2}\left(x_{2}, 0, z_{2}\right)$; then, $\min \left\{\zeta^{+}\left(\alpha_{1}\right), \zeta^{+}\left(\alpha_{2}\right)\right\}=(1 / 2)$ and $\max \left\{\zeta^{-}\left(\alpha_{1}\right), \zeta^{+}\left(\alpha_{2}\right)\right\}=(1 / 2)$. For any $a, b \in \mathbb{R}$,

$$
\begin{aligned}
& \zeta^{+}\left(a \alpha_{1}+b \alpha_{2}\right)=\zeta^{+}\left(a x_{1}+b x_{2}, a y_{1}, b z_{2}\right)=\frac{1}{2} \\
& \zeta^{-}\left(a \alpha_{1}+b \alpha_{2}\right)=\zeta^{-}\left(a x_{1}+b x_{2}, a y_{1}, b z_{2}\right)=0
\end{aligned}
$$

which satisfies the conditions of Definition 11.

Definition 15. Let $X \neq \phi$ be a finite universe. The subset $Q \subseteq Q(X)$ is a subfamily of $q$-ROFSs satisfying

(1) $\phi \in \mathbb{Q}$.

(2) For any $\zeta_{1}=\left(\zeta_{1}^{+}, \zeta_{1}^{-}\right) \in \mathbb{Q}, \zeta_{2}=\left(\zeta_{2}^{+}, \zeta_{2}^{-}\right) \in \mathbb{Q}(X)$ where $\quad 0 \leq\left(\zeta_{1}^{+}(y)\right)^{q}+\left(\zeta_{1}^{-}(y)\right)^{q} \leq 1$, $0 \leq\left(\zeta_{2}^{+}(y)\right)^{q}+\left(\zeta_{2}^{-}(y)\right)^{q} \leq 1, \quad q \geq 1$, and if $\zeta_{2} \subset \zeta_{1}$, then $\zeta_{2} \in \mathbb{Q}$, for all $y \in X$.

(3) For any $\zeta_{1}, \zeta_{2} \in \mathbb{Q}$ and $\left|\sup p\left(\zeta_{1}\right)\right|<\left|\sup p\left(\zeta_{2}\right)\right|$, there exists $\zeta_{3} \in Q$ such that

(a) $\zeta_{1} \subset \zeta_{3} \subseteq \zeta_{1} \cup \zeta_{2}$, for any $y \in X$. (b) $m\left(\zeta_{3}\right) \geq \inf \left\{m\left(\zeta_{1}\right), m\left(\zeta_{2}\right)\right\}, m\left(\zeta_{i}\right)=\inf \left\{\mathcal{S}_{\widetilde{q}}\left(\zeta_{i}\right.\right.$ $\left.(x)) \mid x \in \operatorname{supp}\left(\zeta_{i}\right)\right\}$.

Then, the pair $\mathscr{Q} \mathscr{M}(X)=(X, Q)$ is called $q-$ ROFM and the set $Q$ is the subfamily of all independent $q$-ROFSs of Q. $M(X)$.

Proposition 5. Let $\widetilde{X}=(X, \zeta)$ be a $q$-ROFVS and $Q$ be $a$ subset of $Q(X)$ containing $q-R O F$ linearly independent column vectors in $\tilde{X}$. The pair $(X, Q)$ is a $q$-ROFM on $X$.

Proof. Suppose that $X$ is a non-empty set containing column labels of a $q$-ROF matrix, and $\zeta_{x}$ represents a $q$-ROF submatrix containing those columns which are labeled in $X$. Consider a set $Q$ of $q$-ROF linearly independent column vectors of $\zeta_{x}$, i.e.,

$$
\begin{aligned}
\mathbb{Q}= & \left\{\zeta_{x} \in \mathbb{Q}(X) \mid \text { column vectors of } \zeta_{x} \text { are } q\right. \\
& \text { - rung orthopair fuzzy linearly independent }\} .
\end{aligned}
$$

For any submatrix $\zeta_{x}=\left[a_{i j}\right]_{m \times n}$, we have $\left|\zeta_{x}\right|=\sum_{i=1}^{m} \sup \left\{\xi_{x}\left(a_{i 1}\right), \xi_{x}\left(a_{i 2}\right), \ldots, \xi_{x}\left(a_{i n}\right)\right\}$. It is easy to see from Definitions 11 and 15 that $(X, Q)$ is $\mathcal{O} \mathscr{M}(X)$.

Note that $\sigma \notin \mathbb{Q}$ is called dependent $q$ - ROFS.

Definition 16. Let $Q \mathscr{M}(X)$ be a $q-\mathrm{ROFM}$ and $\mathcal{Q}_{c}$ be a family of dependent $q-$ ROFSs in $Q \mathscr{M}(X)$. The minimal dependent (inclusion wise) set $\sigma \in \mathbb{Q}_{c}$ is called $q$-ROF circuit of $Q \mathscr{M}(X)$ and $\mathcal{Q}_{c}(\mathscr{Q} \mathscr{M})$ represents the subclass of all circuits of $\mathbb{Q} \mathscr{M}$, i.e.,

$$
\mathbb{Q}_{c}\left(Q_{\mathscr{Q}} \mathscr{M}\right)=\left\{\sigma \mid \sigma \in \mathbb{Q}_{c} \text {, inclusion - wise minimal }\right\} .
$$

Note that the elements of $Q_{c}\left(Q_{\mathscr{Q}} \mathscr{M}\right)$ follow the properties:

(1) $\phi \notin \mathbb{Q}_{c}(\mathscr{Q} \mathscr{M})$.

(2) Let $\sigma_{1}$ and $\sigma_{2}$ be $q$-rung orthopair fuzzy circuits with $\sigma_{1} \subseteq \sigma_{2}$; then, $\sup p\left(\sigma_{1}\right)=\sup p\left(\sigma_{2}\right)$.

(3) Let $\sigma_{1}, \sigma_{2} \in \mathbb{Q}_{c}\left(\mathbb{Q}_{\mathscr{M}}\right)$ and $\alpha \in \mathbb{Q}(X)$ with $\alpha(x)=\inf \left\{\sigma_{1}(x), \sigma_{2}(x)\right\}$ where $x \in \sup p\left(\sigma_{1} \cap \sigma_{2}\right)$. Then, there exists $\sigma_{3}$ satisfying

(a) $\sigma_{3} \neq \phi$ and $\sigma_{3} \in Q_{c}(\mathbb{Q} \mathscr{M})$.

(b) $\sigma_{3} \subseteq\left(\sigma_{1} \cup \sigma_{2}\right)-\{(x, \alpha(x))\}$.

Definition 17. Let $Q \mathscr{Q} \mathscr{M}(X)=(X, Q)$ be a $q$-ROFM. Consider an element $\zeta_{i} \in Q$; then, $\zeta_{i}$ is called maximal independent set in a matroid $\mathscr{Q} \mathscr{M}(X)$ if there does not exist $\zeta_{j} \in \mathbb{Q}$ that contains $\zeta_{i}$. A maximal independent set in $\mathbb{Q} \mathscr{M}(X)$ is called $q$-ROF base or basis of $\mathbb{Q} \mathscr{M}(X)$. The collection of all $q$-ROF basis is defined as

$$
\mathscr{B}(\mathscr{P} \mathscr{M})=\{\zeta \mid \zeta \in \mathbb{Q}, \xi \text { is maximal independent in } \mathscr{Q} \mathscr{M}\}
$$

Note that although $q$-ROF basis contains all the independent sets in $Q \mathscr{M}(X)$, there exist some $Q \mathscr{M}(X)$ that do not have $q$-ROF basis. 
Example 2. Let $Q(X)$ be a family of all $q$-ROFSs defined on a non-empty set $X$. Then, for a positive integer $i$ and $|X|=j$ with $i \leq j$, the set $\mathbb{Q}$ is defined as

$$
\mathbb{Q}=\{\zeta \in \mathbb{Q}(X):|\sup p(\zeta)| \leq i\} .
$$

The pair $(X, \mathcal{Q})=(\mathscr{U} \mathscr{F})_{j}^{i}$ is called $q$-ROF uniform matroid. Note that the subfamilies of all $q$-ROFSs of $X$ with the sizes $i+1$ and $i$ are called the $q$-ROF circuits and $q$-ROF basis of $(\mathcal{U F})_{j}^{i}$, respectively.

Definition 18. Let $Q \mathscr{M}(X)$ be a $q$-ROFM. The $q$-ROF rank function $\mathbb{Q}_{R}: \mathbb{Q}(X) \longrightarrow[0, \infty) \times[0, \infty)$ is defined as

$$
\mathbb{Q}_{R}(\mu)=\sup \{|\zeta|: \zeta \subseteq \mu \text { and } \zeta \in \mathbb{Q}\},
$$

where $|\zeta|=\sum_{y \in X}\left(\zeta^{+}(y), \zeta^{-}(y)\right)=\left(\sum_{y \in X} \zeta^{+}(y), \quad \sum_{y \in X} \xi^{-}\right.$ $(y))$. Also, $\left|\zeta_{1}\right| \leq\left|\zeta_{2}\right|$ iff $\sum_{y \in X} \zeta_{1}^{+}(y) \leq \sum_{y \in X} \xi_{2}^{-}(y)$ and $\sum_{y \in X} \zeta_{1}^{-}(y) \geq \sum_{y \in X} \zeta_{2}^{-}(y)$.

It is observed from definition that the $q$-ROF rank function follows the following properties:

(1) If $\zeta \in \mathbb{Q}(X)$, then $\mathbb{Q}_{R}(\zeta)=|\zeta|$.

(2) If $\zeta \in \mathbb{Q}(X)$, then $Q_{R}(\zeta) \leq|\zeta|$.

(3) If $\zeta_{1}, \zeta_{2} \in \mathbb{Q}(X)$ and $\xi_{1} \subseteq \zeta_{2}$, then $\mathbb{Q}_{R}\left(\zeta_{1}\right) \leq Q_{R}\left(\zeta_{2}\right)$.

Definition 19. Let $\left\{q_{1}, q_{2}, \ldots, q_{n}\right\}$ be a set of " $n$ " $q$-ROFNs. Then, the pair $q_{i}=\left(q_{i}^{\prime}, s_{i}^{\prime}\right)$ satisfies the ordering for each $1 \leq i \leq n$ :

$$
q_{i}^{\prime} \leq q_{i+1}^{\prime}, s_{i}^{\prime} \geq s_{i+1}^{\prime} \Longleftrightarrow q_{i} \leq q_{i+1} .
$$

We say $q$ instead of $q=\left(q^{\prime}, s^{\prime}\right)$ and $\mathbf{0} \leq q \leq \mathbf{1}$, where $\mathbf{0}=$ $(0,1)$ and $\mathbf{1}=(1,0)$ with $\mathcal{\delta}_{\widetilde{q}}(\mathbf{0})=0$ and $\mathcal{S}_{\widetilde{q}}(\mathbf{1})=1$, respectively.

Definition 20. The $q$-cut level set for $\mathbf{0}<q \leq \mathbf{1}$ of a $q$-ROFS $\zeta \in \mathbb{Q}(X)$ is a crisp set which is defined as follows:

$$
\mathbb{Q}_{q}(\zeta)=\left\{x \in X: \zeta^{+}(x) \geq q^{\prime} \text { and } \zeta^{-}(x) \leq s^{\prime}\right\} .
$$

Theorem 1. Let $\mathbb{Q} \mathscr{M}(X)=(X, \mathbb{Q})$ be a $q$-ROFM and $\mathbb{Q}_{q}$ be a collection of all $q$-cut levels of $q-R O F$ independent sets where $\mathbf{0}<q \leq \mathbf{1}$, i.e.,

$$
\mathbb{Q}_{q}=\left\{Q_{q}(\xi) \mid \xi \in Q\right\}
$$

Then, $\mathscr{M}_{q}=\left(X, Q_{q}\right)$ is a crisp matroid on $X$.

Proof. The proof is very straight forward from Definition 20, and $Q_{q}$ is a collection of crisp subsets of $X$. Then, for each $\mathbf{0}<q \leq \mathbf{1}$, we have $\mathscr{M}_{q}=\left(X, Q_{q}\right)$.

Definition 21. Let $X \neq \phi$ be a finite universe and $Q \mathscr{M}(X)$ be a $q$-ROFM. Then, we have a finite sequence $q_{1}<q_{2}<\cdots<q_{n}$ such that

(1) $q_{0}=\mathbf{0}, q_{n} \leq \mathbf{1}$.

(2) $Q_{r}$ is non - empty, if $\mathbf{0}<r \leq q_{n}$, and $Q_{r}$ is empty, if $r$ $>q_{n}$.
(3) If $q_{i}<r_{1}, r_{2}<q_{i+1}$, then $Q_{r_{1}}=Q_{r_{2}}, 0 \leq i \leq n-1$.

(4) If $q_{i}<r_{1}<q_{i+1}<r_{2}<l_{i+2}$, then $Q_{r_{1}} \supset Q_{r_{2}}, 0 \leq i \leq n-2$.

The sequence $q_{0}=\mathbf{0}, q_{1}, q_{2}, \ldots, q_{n}$ is called fundamental sequence of $\mathcal{Q} \mathscr{M}(X)$.

Corollary 1. From Theorem 1 and Definition 21 , for $1 \leq i \leq n$, assume that $\bar{q}_{i}=(1 / 2)\left(q_{i-1}+q_{i}\right)$; then, $\mathscr{M}_{\bar{q}_{1}} \supset \mathscr{M}_{\bar{q}_{2}} \supset \cdots \supset \mathscr{M}_{\bar{q}_{n}}$ is called a $\mathscr{M}_{q}$-induced matroid sequence.

Theorem 2. Let $\mathbf{0}=q_{0}<q_{1}<q_{2}<\cdots<q_{n} \leq \mathbf{1}$ be a finite fundamental sequence and $\left(X, Q_{q_{1}}\right),\left(X, Q_{q_{2}}\right), \ldots,\left(X, Q_{q_{n}}\right)$ be finite sequence of crisp matroids regarding this fundamental sequence. For each $q_{i-1}<q \leq q_{i}(i=1,2, \ldots, n)$, we assume $Q_{q}=Q_{q_{i}}$ and for $q_{n}<q \leq 1, Q_{q}=\phi$. Then the pair $(X, \widehat{Q})$ is a $q-R O F M$ where $\widehat{Q}$ is defined as

$$
\widehat{Q}=\left\{\zeta \in \mathbb{Q}(X) \mid Q_{q}(\zeta) \in \mathbb{Q}_{q}, \mathbf{0}<q \leq \mathbf{1}\right\} .
$$

Proof. It is easy to see that $\phi \in \widehat{Q}$ as $\mathbb{Q}_{q}=\phi$ for $q_{n}<q \leq \mathbf{1}$. Now, assume that $\zeta_{1} \in \widehat{Q}$, and $\zeta_{2} \in \mathbb{Q}(X)$ such that $\zeta_{2} \subseteq \zeta_{1}$. It is clear from definition of $\widehat{Q}$ that for each $\mathbf{0}<q \leq \mathbf{1}$, $Q_{q}\left(\zeta_{1}\right) \in Q_{q}$, so $Q_{q}\left(\zeta_{2}\right) \subseteq Q_{q}\left(\zeta_{1}\right)$, and since we have that $\left(X, Q_{q}\right)$ is a crisp matroid for $Q_{q}$, it means $Q_{l}\left(\zeta_{2}\right) \in Q_{l}$, and hence $\zeta_{2} \in \widehat{Q}$ and proves (18) of Definition 15. Now, let $\zeta_{1}, \zeta_{2} \in \mathbb{Q}$ with $\left|\sup p\left(\zeta_{2}\right)\right|<\left|\sup p\left(\zeta_{1}\right)\right|$. It is known that $m\left(\zeta_{i}\right)=\inf \left\{\mathcal{S}_{\widetilde{q}}\left(\zeta_{i}(x)\right): x \in \sup p\left(\zeta_{i}\right)\right\}$. Let $\zeta$ be a $q-\mathrm{ROFN}$ defined as

$$
\eta=\min \left\{m\left(\zeta_{1}\right), m\left(\zeta_{2}\right)\right\}
$$

It is easy to observe from definition of $\eta$ that $\mathbb{Q}_{\eta}$ contains the support of both $\zeta_{1}$ and $\zeta_{2}$. Note that $Q_{\eta}$ contains independent subsets; then, there exists an independent subset $I \in Q_{\eta}$ satisfying

(1) $I$ contains $\sup p\left(\zeta_{2}\right)$, for all $x \in X$.

(2) $I \subseteq \sup p\left(\zeta_{1}\right) \cap \sup p\left(\zeta_{2}\right)$.

Let us define $\zeta_{3}$ as

$$
\zeta_{3}(y)= \begin{cases}\eta, & y \in I-\left\{\sup p\left(\zeta_{2}\right)\right\}, \\ \zeta_{2}(y), & y \in \sup p\left(\zeta_{2}\right), \\ \mathbf{0}=(0,1), & \text { otherwise }\end{cases}
$$

which shows that $\zeta_{3}$ is $q$ - ROFS and satisfies (20) of Definition 15 . Hence, $(X, \widehat{Q})$ is $q-$ ROFM.

Theorem 3. Let $Q \mathscr{M}=(X, Q)$ be a $q-R O F M$ and from Definition 20 and $Q_{q}$ defined in Theorem 1 , for each $\mathbf{0}<q \leq \mathbf{1}$, $\mathscr{M}_{q}=\left(X, Q_{q}\right)$ is a crisp matroid. Let $\widehat{\mathbb{Q}} \stackrel{q}{=}\left\{\zeta \in \mathscr{Q}(X) \mid Q_{q}(\zeta) \in \mathbb{Q}_{q}, \mathbf{0}<l \leq \mathbf{1}\right\}$. Then, $\mathbb{Q}=\widehat{Q}$.

Proof. It is easy to deduce from definition of $\widehat{Q}$ that $Q \subset \widehat{Q}$. For $\zeta \in \widehat{Q}$, let $\left\{v_{i}\right\}_{i=1}^{t}$ be a non-zero $q$-rung orthopair fuzzy range with $v_{i}=\left(v_{i}^{*}, v_{i}^{\prime}\right)$ and order $v_{1}>v_{2}>\cdots>v_{t}>0$. One can notice that for each $1 \leq i \leq t$ and $\zeta \in \widehat{Q}$, we have that $Q_{v_{i}}(\zeta) \in Q_{v_{i}}$. So, if $1 \leq i \leq t-1$, then from Definition 21, $\widehat{Q}_{v_{i}}(\zeta) \subset \mathbb{Q}_{v_{i+1}}(\zeta)$. To prove $\widehat{Q} \subset Q$, we define a $q$-rung orthopair fuzzy set $\varphi \in \mathbb{Q}(X)$ for each $1 \leq i \leq t$ and $u \in X$ as 


$$
\varphi_{i}(u)= \begin{cases}v_{i}=\left(v_{i}^{*}, v_{i}^{\prime}\right), & \text { if u is in } q-\text { cut for } \zeta, \\ \mathbf{0}=(0,1), & \text { otherwise, }\end{cases}
$$

with $0<\left(\nu^{*}\right)^{q}+\left(\nu^{\prime}\right)^{q} \leq 1$. Since we have $\mathbb{Q}_{v_{i}}(\zeta) \in \mathbb{Q}_{v_{i}}, \cup_{i} \varphi_{i}=$ $\zeta$ for $1 \leq i \leq t$ that gives $\varphi_{i} \in \mathbb{Q}$. Here to show $\zeta \in$, we use induction process. For each $1 \leq i \leq t$, we consider

$$
\left\{\begin{array}{l}
\sup p\left(\varphi_{i}\right)=\left\{u_{k}\right\}_{k=1}^{n_{i}} \\
\sup p(\zeta)=\left\{u_{j}\right\}_{j=1}^{n_{t}}
\end{array}\right.
$$

Since $\varphi_{1} \in \mathbb{Q}$ is an independent $q$-ROFS, it is enough to show that if $\cup_{i=1}^{l-1} \varphi_{i} \in \mathbb{Q}$ for $r>l-1$, then for each $r>l$, we have $\cup_{i=1}^{l} \varphi_{i} \in \mathbb{Q}$. Define

$$
\Phi_{1}(u)= \begin{cases}v_{l}=\left(v_{l}^{*}, v_{l}^{\prime}\right), & \text { if } u \in\left\{u_{1}, u_{2}, \ldots, u_{n_{l-1}+1}\right\}, \\ \mathbf{0}=(0,1), & \text { otherwise }\end{cases}
$$

which shows that for each $1<i<l-1, v_{i}>v_{l}$, so $\Phi_{1} \subset \varphi_{l}$ and $\Phi_{1} \in \mathbb{Q}$. Define another $q$-ROFS $\lambda$ as

$$
\Psi_{1}(u)= \begin{cases}\zeta\left(u_{n_{l-1}+1}\right)=\left(v_{l}^{*}, v_{l}^{\prime}\right), & \text { if } u_{n_{l-1}+1}, \\ (0,1), & \text { otherwise. }\end{cases}
$$

Using the induction method, we have $\sup p\left(\cup_{i=1}^{l-1} \varphi_{i}\right)=$ $\left\{u_{1}, u_{2}, \ldots, u_{n_{l-1}}\right\}$ where $\cup_{i=1}^{l-1} \varphi_{i}$ is an independent set, and $\left\{m\left(\cup_{i=1}^{l-1} \varphi_{i}\right)\right\}>v_{l}$. So, from Definition 15, $\cup_{i=1}^{l} \varphi_{i} \cup \Psi_{1} \in \mathbb{Q}$ is independent in Q.M. If $n_{l-1}+1=n_{l}$, then we have that $\cup_{i=1}^{l} \varphi_{i}$ is also an independent set in Q.M. But, if $n_{l-1}+1<n_{l}$, then to move further, we define $q$-ROFS as

$$
\Phi_{2}(u)= \begin{cases}\left(v_{l}^{*}, v_{l}^{\prime}\right), & \text { if } u \in\left\{u_{1}, u_{2}, \ldots, u_{n_{l-1}+1}\right\} \cup\left\{u_{n_{l-1}+2}\right\}, \\ (0,1), & \text { otherwise. }\end{cases}
$$

From Definition 19, $\Phi_{2} \in \mathbb{Q}(X)$, and from $\Phi_{2} \subset \varphi_{l}$, $\Phi_{2} \in \mathbb{Q}$ is an independent set in $\mathcal{Q} \mathscr{M}$. Similarly, define another $q$-ROFS as

$$
\Psi_{2}(u)= \begin{cases}\zeta\left(u_{n_{l-1}+2}\right)=\left(v_{l}^{*}, v_{l}^{\prime}\right), & \text { if } u_{n_{l-1}+2} \\ (0,1), & \text { otherwise. }\end{cases}
$$

Since $\sup p\left(\cup_{i=1}^{l-1} \varphi_{i} \cup \Psi_{1}\right)=\left\{u_{1}, u_{2}, \ldots, u_{n_{l-1}}\right\} \cup\left\{u_{n_{l-1}+1}\right\}$ and $\left(\cup_{i=1}^{l-1} \varphi_{i} \cup \Psi_{1}\right)>\nu_{l}$, then again from Definition 15 , $\cup_{i=1}^{l-1} \varphi_{i} \cup \Psi_{1} \cup \Psi_{2}$ is an independent set in Q.M. So, $\cup_{i=1}^{l-1} \varphi_{i}$ is also an independent set in $Q \mathscr{M}$ for $n_{l-1}+1=n_{l}$. But, if $n_{l-1}+1<n_{l}$, then to proceed further, we obtain a new $q$-ROFS $\chi_{n}=\cup_{i=1}^{l-1} \varphi_{i} \cup \Psi_{1} \cup \Psi_{2} \cdots \cup \Psi_{n}$ and hence $\chi_{n}=\cup_{i=1}^{l-1} \varphi_{i}$ is an independent set in $Q \mathscr{M}$.

The next result is the direct consequence of Theorem 1.4 discussed in [34].

Corollary 2. Let $Q \mathscr{M}=(X, Q)$ be a $q-R O F M$ and $\zeta \in \mathbb{Q}(X)$. Then, $\zeta \in \mathbb{Q}(X)$ if and only if for each $q \in \mathscr{R}^{+}(\zeta)$ we have $\mathbb{Q}_{q}(\zeta) \in \mathbb{Q}_{q}$.

Theorem 4. Let $\zeta \in Q$ be a q-rung orthopair fuzzy base of a $q-\operatorname{ROFM}(X, Q)$. Then, for each $x \in X, \varrho_{\zeta}(x)=0$.
Proof. Suppose on contrary that $x_{i} \in X$ with $\varrho_{\zeta}\left(x_{i}\right)=\alpha>0$ and take $\alpha^{*}=(\alpha / 2)$. Let $\zeta^{\prime} \in \mathbb{Q}(X)$ such that, for each $x \in X, \delta_{\widetilde{q}}\left(\zeta^{\prime}\right)=\mathcal{S}_{\widetilde{q}}(\zeta)$. Define

$$
\varrho_{\zeta^{\prime}}(x)= \begin{cases}\varrho_{\zeta}(x), & \text { if } x \in X \text { and } x \neq x_{i}, \\ \alpha^{*}, & \text { otherwise. }\end{cases}
$$

$\mathscr{A}_{\tilde{q}}(\zeta)=\mathscr{A}_{\tilde{q}}\left(\zeta^{\prime}\right)$, which show that $\zeta<\zeta^{\prime}$. But, for each $q \in \mathscr{R}^{+}(\zeta)$, we have $Q_{q}(\zeta)=Q_{q}\left(\zeta^{\prime}\right)$. Hence, it is easy to see from Corollary 2 that $\zeta^{\prime} \in \mathbb{Q}$, which gives the contradiction and completes the proof.

Theorem 5. Let $0=q_{0}<q_{1}<q_{2} \cdots<q_{n} \leq 1$ be a fundamental sequence of $q-R O F M Q \mathscr{Q}=(X, Q)$. Let $\zeta=\left(\zeta^{+}, \zeta^{-}\right)$be a q-rung orthopair fuzzy basis of QMM; then,

$$
\mathscr{R}^{+}\left(\zeta^{+}, \zeta^{-}\right) \subseteq\left\{q_{1}, q_{2}, \ldots, q_{n}\right\} .
$$

Proof. Let $\left(\zeta_{i}^{+}, \zeta_{i}^{-}\right)$be a $q$-run orthopair fuzzy basis. So, $\left(\zeta_{i}^{+}, \zeta_{i}^{-}\right) \in Q$. For each $q \in \mathscr{R}^{+}\left(\zeta_{i}^{+}, \zeta_{i}^{-}\right)$, we have $Q_{q}\left(\zeta_{i}^{+}, \zeta_{i}^{-}\right)$. Suppose that there exists $t \in \mathscr{R}^{+}\left(\zeta_{i}^{+}, \zeta_{i}^{-}\right)$with $q_{i}<t<q_{i+1}$ and $\lambda=\left(q_{i+1}-t\right) / 2$. Assume that a $q$-rung orthopair fuzzy set $\zeta_{j}=\left(\zeta_{j}^{+} \zeta_{j}^{-}\right)$is an elementary $q$-ROFS, defined with the $\sup p\left(\zeta_{j}^{+}, \zeta_{j}^{-}\right)=Q_{t}\left(\zeta_{i}^{+}, \zeta_{i}^{-}\right)$and $\mathscr{R}^{+}\left(\zeta_{j}^{+}, \zeta_{j}^{-}\right)=\left(t+q_{i+1}\right) / 2$. Take $\left(\zeta_{k}^{+}, \zeta_{k}^{-}\right)=\left(\zeta_{i}^{+}, \zeta_{i}^{-}\right) \vee\left(\zeta_{j}^{+}, \zeta_{j}^{-}\right)$and for each $0<q \leq 1$, if $t<q<\left(t+q_{i+1}\right) / 2$, then $\mathbb{Q}_{q}\left(\zeta_{k}^{+}, \zeta_{k}^{-}\right)=\mathbb{Q}_{t}\left(\zeta_{i}^{+}, \zeta_{i}^{-}\right) \in \mathbb{Q}_{t}$; otherwise, $\mathbb{Q}_{q}\left(\zeta_{k}^{+}, \zeta_{k}^{-}\right)=\mathbb{Q}_{q}\left(\zeta_{i}^{+}, \zeta_{i}^{-}\right) \in \mathbb{Q}_{q}$. By Corollary 2, $\left(\zeta_{k}^{+}, \zeta_{k}^{-}\right) \in Q$. So, for $\left(\zeta_{i}^{+}, \zeta_{i}^{-}\right)$, there exists $x_{r} \in \sup p\left(\zeta_{i}^{+}, \zeta_{i}^{-}\right)$ with $\mathcal{S}_{\widetilde{q}}\left(\zeta_{i}^{+}\left(x_{r}\right), \zeta_{i}^{-}\left(x_{r}\right)\right)=t$, and hence $\mathcal{S}_{\widetilde{q}}\left(\zeta_{k}^{+}\right.$ $\left.\left(x_{r}\right), \zeta_{k}^{-}\left(x_{r}\right)\right)=\left(t+q_{i+1}\right) / 2$, which contradicts that $\left(\zeta_{i}^{+}, \zeta_{i}^{-}\right)$ is maximal and a $q$-rung orthopair fuzzy basis.

\section{Application}

4.1. Case Study: Human Trafficking. With a rapid increase in the population of different continents, including Asia and Africa, it is unable to stop human trafficking. Many reasons are behind this, such as poverty, greed for a handsome job, unemployment, low literacy rate, labor, and sexual exploitation. Studies show that millions of people are trapped in this modern slavery, and it is a dilemma that people do not realize they are getting trapped due to unawareness. Still, we are unable to get exact statistics due to unreported cases of human trafficking. An important application is to study human trafficking in different countries and provide a guess about a more suitable way or path that the traffickers can use.

Consider $n$ and $m$ number of countries, say $\left\{C_{1}, C_{2}, \ldots, C_{n}\right\}$ and $\left\{C_{1}^{\prime}, C_{2}^{\prime}, \ldots, C_{m}^{\prime}\right\}$, respectively. The traffickers will move from $C_{1}$ to any of the other $C_{i}$ countries. However, $C_{1}$ is not connected with other $C_{i}$ 's directly or the human trafficking ratio is almost ignorable between them due to multiple factors. The traffickers move in groups and collect their other victims from the countries $C_{j}^{\prime}$ where $j=1,2, \ldots, m$, to move any one of the countries $C_{i}$ where $i=2,3, \ldots, n$. If $C_{1}$ is the starting point, they will definitely move to any of the other $C_{i}$ (where $i=2, \ldots, n$ ) for their next station. The state agencies seek to solve this puzzle used by 
(1) Input:

(i) Consider two finite sets of countries $\left\{C_{1}, C_{2}, \ldots, C_{n}\right\}$ and $\left\{C_{1}^{\prime}, C_{2}^{\prime}, \ldots, C_{m}^{\prime}\right\}$, and each $C_{i}$ and $C_{j}^{\prime}$ mark a vertex of the graph $G$.

(ii) Mark all the edges and give the direction between the countries regarding given $q$-rung orthopair fuzzy information and given problem, that is, for each $1 \leq i \leq m n, a_{i}\left(\zeta_{i}^{+}, \zeta_{i}^{-}\right)$represents a directed edge that represents the flow between two countries. There is no edge between any of the $C_{i}$ 's and also no edge between $C_{j}$ 's.

(2) Calculate the score function $\mathcal{S}_{\tilde{q}}\left(a_{i}\right)=(1 / 2)\left(1+\left(\zeta_{i}^{+}\right)^{q}-\left(\zeta_{i}^{-}\right)^{q}\right)$.

(3) Find all the edge sets of length $m n-1$ and remove all the cycles of length $m n-1$.

(4) Find $B=\left\{X_{l}=\left\{a_{i_{k}}\right\}_{k=1}^{m+n-1} \mid X_{l}\right.$ is maximal independent $\}$, where $1 \leq i \leq m n$ and $l=1,2, \ldots, n^{m} m^{n}$.

(5) Determine $B^{\prime}$ by removing all $X_{l}$ from $B$ such that $X_{l}$ 's are not spanning paths (the path runs through each vertex of the graph exactly once).

(6) Reduce the set $B^{\prime}$ to $B^{\prime \prime}$ by removing all the spanning paths from $B^{\prime}$ which are not the cases according to the directions given in the

(7) Compute $\overline{T_{i}}=\left\{\sum\left\{\mathcal{S}_{\tilde{q}}\left(a_{i_{k}}\right)\right\}_{k=1}^{n-1}\right\}$ where each $\left\{a_{i_{k}}\right\}_{k=1}^{n-1} \in B^{\prime \prime}$.
(8) Find $\max \left(\overline{T_{i}}\right)$.

Output: in the last step, the heaviest path will help us to take suitable measures according to flow of human trafficking given in the data.

Algorithm 1: Selection of the heaviest path.

TABle 1: 3-Rung orthopair fuzzy information of human trafficking between regions from Figure 1.

\begin{tabular}{lccc}
\hline Serial no. & Connections & $a_{i}\left(\xi_{i}^{+}, \xi_{i}^{-}\right)$ & $\mathcal{S} \sim\left(\zeta^{+}(x), \zeta^{-}(x)\right)$ \\
\hline 1 & $V_{3} \longrightarrow V_{1}$ & $(0.33,0.04)$ & 0.6796 \\
2 & $V_{3} \longrightarrow V_{2}$ & $(0.25,0.05)$ & 0.5077 \\
3 & $V_{4} \longrightarrow V_{1}$ & $(0.26,0.5)$ & 0.4462 \\
4 & $V_{2} \longrightarrow V_{4}$ & $(0.13,0.02)$ & 0.5010 \\
5 & $V_{1} \longrightarrow V_{5}$ & $(0.04,0.1)$ & 0.4995 \\
6 & $V_{5} \longrightarrow V_{2}$ & $(0.8,0.03)$ & 0.7559 \\
\hline
\end{tabular}
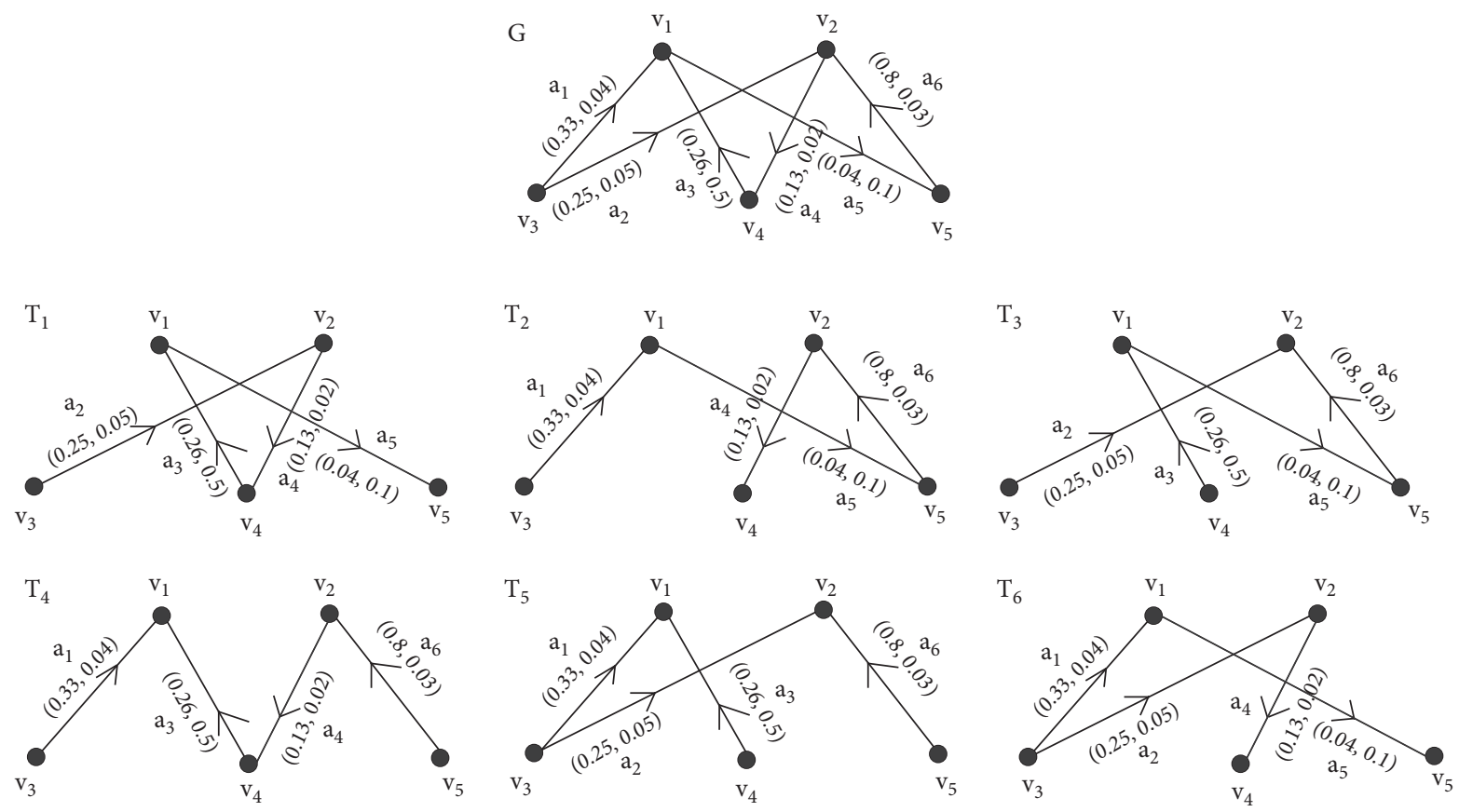

Figure 1: q-Rung orthopair fuzzy graph representation of human trafficking data.

the traffickers for trafficking across different stations. The procedure to track their way or path regarding given information is described in Algorithm 1.
We take some data to find the illegal immigration routes used by traffickers for human trafficking. This model is in the form of directed fuzzy graphs. The edge between the two 
TABLE 2: Directed spanning paths with the sum of their score functions and weights.

\begin{tabular}{lccc}
\hline Serial no. & $T_{i}=\left\{a_{i}\right\}$ & $\sum\left\{\mathcal{S} \sim_{3}\left(a_{i}\right)\right\}$ & $\overline{T_{i}}$ \\
\hline 1 & $\left\{a_{2}, a_{3}, a_{4}, a_{5}\right\}$ & $\{0.5077+0.4462+0.5010+0.4995\}$ & 1.9544 \\
2 & $\left\{a_{1}, a_{4}, a_{5}, a_{6}\right\}$ & $\{0.6796+0.5010+0.4995+0.7559\}$ & 2.436 \\
\hline
\end{tabular}

countries is represented by the $q$-rung fuzzy information. The membership part shows the ratio of human trafficking from one country to another. The non-membership part shows parameters of measurements taken by the concerned governments to stop human trafficking (government response action to reduce flow). We use the data from the model given by Mordeson and Mathew [45]. The data of illegal human flow between different regions are given in Table 1 [45]. In this study, we consider five regions: the Middle East $\left(V_{1}\right)$, West Central Europe $\left(V_{2}\right)$, East Asia and Pacific $\left(V_{3}\right)$, West Southern Europe $\left(V_{3}\right)$, North America $\left(V_{4}\right)$, and Central America $\left(V_{5}\right) . V=\left\{V_{1}, V_{2}, V_{3}, V_{4}, V_{5}\right\}$ and $E=\left\{a_{1}, a_{2}, a_{3}, a_{4}, a_{5}, a_{6}\right\}$ represent the set of vertices and directed edges of graph $G$, respectively (see Figure 1 ). We can analyze the flow of human trafficking by these directed edges. Our task is to find a frequently used path by the traffickers so that relevant governments can take some measures to stop this abuse. We calculate the score functions of all the given $q$-ROF information (see Table 1 ). To engage all the five concerned governments, it is easy to see from Figure 1 that four edges need to pass through all vertices, and such total possibilities are 15. However, cycles of length four cannot be the choice while solving such a puzzle. The remaining 12 edge sets are maximal independent sets that can be useful to find the most used path by the traffickers. We say $B$, i.e., $B=\left\{\left\{a_{1}, a_{2}, a_{3}, a_{5}\right\}, \quad\left\{a_{1}, a_{2}, a_{3}, a_{6}\right\}\right.$, $\left\{a_{1}, a_{2}, a_{4}, a_{5}\right\},\left\{a_{1}, a_{2}, a_{4}, a_{6}\right\},\left\{a_{2}, a_{3}, a_{4}, a_{5}\right\},\left\{a_{2}, a_{3}, a_{5}, a_{6}\right\}$, $\left\{a_{2}, a_{3}, a_{4}, a_{6}\right\},\left\{a_{2}, a_{4}, a_{5}, a_{6}\right\},\left\{a_{1}, a_{3}, a_{4}, a_{5}\right\},\left\{a_{1}, a_{3}, a_{5}, a_{6}\right\}$, $\left.\left\{a_{1}, a_{4}, a_{5}, a_{6}\right\},\left\{a_{1}, a_{3}, a_{4}, a_{6}\right\}\right\}$. In the next step, the set $B$ reduces to $B^{\prime}$ by removing maximal independent sets which are not spanning paths, i.e., $B^{\prime}=\left\{a_{1}, a_{2}, a_{3}, a_{6}\right\}$, $\left\{a_{1}, a_{2}, a_{4}, a_{5}\right\},\left\{a_{2}, a_{3}, a_{4}, a_{5}\right\},\left\{a_{2}, a_{3}, a_{5}, a_{6}\right\},\left\{a_{1}, a_{4}, a_{5}, a_{6}\right\}$, $\left.\left\{a_{1}, a_{3}, a_{4}, a_{6}\right\}\right\}$. However, the graph shown in Figure 1 is directed, so four more maximal independent sets are deleted due to absurdity in these cases. So, only $T_{1}=\left\{a_{2}, a_{3}, a_{4}, a_{5}\right\}$ and $T_{2}=\left\{a_{1}, a_{4}, a_{5}, a_{6}\right\}$ are the cases. Table 2 shows that $T_{2}=\left\{a_{1}, a_{4}, a_{5}, a_{6}\right\}$ is the heaviest path with the score function 2.436. We find that given five countries should increase their government response action against this obtained path used frequently by the traffickers. They should take some measures to minimize human trafficking.

\section{Comparison of Given Model with Intuitionistic and Pythagorean Fuzzy Models}

IFSs and PFSs are known to be the special cases of $q$-ROFSs. The IFS was first introduced by Atanassov [3], and then PFS was discussed later by Yager [15]. The constructions of IFSs and PFSs show the importance of membership and nonmembership functions in various real-life problems. However, there is a limitation in these models; that is, they fail to solve decision-making problems having information in
TABLE 3: Fuzzy information of connections between cities and their score functions (see Figure 2 in [43]).

\begin{tabular}{lcccccc}
\hline Serial no. Connections & $a_{i}\left(\xi_{i}^{+}, \xi_{i}^{-}\right)$ & $\mathcal{\delta}_{2}$ & $\mathcal{S}_{3}$ & $\mathcal{S}_{4}$ & $\mathcal{\delta}_{\tilde{\sim}}$ \\
\hline 1 & $F \longleftrightarrow L$ & $(0.3,0.4)$ & 0.465 & 0.481 & 0.4912 & 0.4960 \\
2 & $F \longleftrightarrow M$ & $(0.7,0.5)$ & 0.62 & 0.609 & 0.5888 & 0.5684 \\
3 & $M \longleftrightarrow N$ & $(0.6,0.7)$ & 0.435 & 0.436 & 0.4447 & 0.4548 \\
4 & $N \longleftrightarrow L$ & $(0.8,0.2)$ & 0.8 & 0.752 & 0.7040 & 0.6636 \\
5 & $F \longleftrightarrow N$ & $(0.6,0.2)$ & 0.66 & 0.604 & 0.5640 & 0.5387 \\
6 & $L \longleftrightarrow M$ & $(0.5,0.7)$ & 0.38 & 0.391 & 0.4112 & 0.4315 \\
\hline
\end{tabular}

TABLE 4: Spanning paths and sum of the score functions of their entries when $q=2$.

\begin{tabular}{llcc}
\hline Serial no. & $T_{i}=\left\{a_{i}\right\}$ & $\sum\left\{\mathcal{S}_{2}\left(a_{i}\right)\right\}$ & $\overline{T_{i}}$ \\
\hline 1 & $\left\{a_{1}, a_{2}, a_{3}\right\}$ & $\{0.465+0.62+0.435\}$ & 1.52 \\
2 & $\left\{a_{1}, a_{2}, a_{4}\right\}$ & $\{0.465+0.62+0.8\}$ & 1.885 \\
3 & $\left\{a_{1}, a_{3}, a_{4}\right\}$ & $\{0.465+0.435+0.8\}$ & 1.7 \\
4 & $\left\{a_{1}, a_{3}, a_{5}\right\}$ & $\{0.465+0.435+0.66\}$ & 1.56 \\
5 & $\left\{a_{1}, a_{3}, a_{6}\right\}$ & $\{0.465+0.435+0.38\}$ & 1.28 \\
6 & $\left\{a_{1}, a_{5}, a_{6}\right\}$ & $\{0.465+0.66+0.38\}$ & 1.505 \\
7 & $\left\{a_{2}, a_{3}, a_{4}\right\}$ & $\{0.62+0.435+0.8\}$ & 1.855 \\
8 & $\left\{a_{2}, a_{4}, a_{5}\right\}$ & $\{0.62+0.8+0.66\}$ & 2.08 \\
9 & $\left\{a_{2}, a_{4}, a_{6}\right\}$ & $\{0.62+0.8+0.38\}$ & 1.8 \\
10 & $\left\{a_{2}, a_{5}, a_{6}\right\}$ & $\{0.62+0.66+0.38\}$ & 1.66 \\
11 & $\left\{a_{3}, a_{5}, a_{6}\right\}$ & $\{0.435+0.66+0.38\}$ & 1.475 \\
12 & $\left\{a_{4}, a_{5}, a_{6}\right\}$ & $\{0.8+0.66+0.38\}$ & 1.84 \\
\hline
\end{tabular}

TABLE 5: Spanning paths and sum of the score functions of their entries when $q=3$.

\begin{tabular}{lccc}
\hline Serial no. & $T_{i}=\left\{a_{i}\right\}$ & $\sum\left\{\underset{\mathcal{S} \sim}{\sim}\left(a_{i}\right)\right\}$ & $\overline{T_{i}}$ \\
\hline 1 & $\left\{a_{1}, a_{2}, a_{3}\right\}$ & $\{0.481+0.609+0.436\}$ & 1.526 \\
2 & $\left\{a_{1}, a_{2}, a_{4}\right\}$ & $\{0.481+0.609+0.752\}$ & 1.842 \\
3 & $\left\{a_{1}, a_{3}, a_{4}\right\}$ & $\{0.481+0.436+0.752\}$ & 1.669 \\
4 & $\left\{a_{1}, a_{3}, a_{5}\right\}$ & $\{0.481+0.436+0.604\}$ & 1.521 \\
5 & $\left\{a_{1}, a_{3}, a_{6}\right\}$ & $\{0.481+0.436+0.391\}$ & 1.308 \\
6 & $\left\{a_{1}, a_{5}, a_{6}\right\}$ & $\{0.481+0.604+0.391\}$ & 1.476 \\
7 & $\left\{a_{2}, a_{3}, a_{4}\right\}$ & $\{0.609+0.436+0.752\}$ & 1.797 \\
8 & $\left\{a_{2}, a_{4}, a_{5}\right\}$ & $\{0.609+0.752+0.604\}$ & 1.965 \\
9 & $\left\{a_{2}, a_{4}, a_{6}\right\}$ & $\{0.609+0.752+0.391\}$ & 1.752 \\
10 & $\left\{a_{2}, a_{5}, a_{6}\right\}$ & $\{0.609+0.604+0.391\}$ & 1.604 \\
11 & $\left\{a_{3}, a_{5}, a_{6}\right\}$ & $\{0.436+0.604+0.391\}$ & 1.431 \\
12 & $\left\{a_{4}, a_{5}, a_{6}\right\}$ & $\{0.752+0.604+0.391\}$ & 1.745 \\
\hline
\end{tabular}

which the sum or sum of the squares of membership and nonmembership values is greater than 1 . To overcome these issues in more complicated information, $q$-ROFSs were introduced by Yager [15]. After introducing vector spaces and matroids based on PFSs in [43], in this study, we propose $q$-ROFVSs and $q$-ROFMs. This section provides the comparative analysis with PFMs and Algorithm 1 discussed 
TABLE 6: Spanning paths and sum of the score functions of their entries when $q=4$.

\begin{tabular}{lccc}
\hline Serial no. & $T_{i}=\left\{a_{i}\right\}$ & $\sum\left\{\mathcal{S}_{2}\left(a_{i}\right)\right\}$ & $\overline{T_{i}}$ \\
\hline 1 & $\left\{a_{1}, a_{2}, a_{3}\right\}$ & $\{0.4912+0.5888+0.4447\}$ & 1.5247 \\
2 & $\left\{a_{1}, a_{2}, a_{4}\right\}$ & $\{0.4912+0.5888+0.704\}$ & 1.784 \\
3 & $\left\{a_{1}, a_{3}, a_{4}\right\}$ & $\{0.4912+0.4447+0.704\}$ & 1.6399 \\
4 & $\left\{a_{1}, a_{3}, a_{5}\right\}$ & $\{0.4912+0.4447+0.7040\}$ & 1.4999 \\
5 & $\left\{a_{1}, a_{3}, a_{6}\right\}$ & $\{0.4912+0.4447+0.4112\}$ & 1.3471 \\
6 & $\left\{a_{1}, a_{5}, a_{6}\right\}$ & $\{0.4912+0.564+0.4112\}$ & 1.4664 \\
7 & $\left\{a_{2}, a_{3}, a_{4}\right\}$ & $\{0.5888+0.4447+0.704\}$ & 1.7375 \\
8 & $\left\{a_{2}, a_{4}, a_{5}\right\}$ & $\{0.5888+0.704+0.564\}$ & 1.8568 \\
9 & $\left\{a_{2}, a_{4}, a_{6}\right\}$ & $\{0.5888+0.704+0.4112\}$ & 1.704 \\
10 & $\left\{a_{2}, a_{5}, a_{6}\right\}$ & $\{0.5888+0.564+0.4112\}$ & 1.564 \\
11 & $\left\{a_{3}, a_{5}, a_{6}\right\}$ & $\{0.4447+0.564+0.4112\}$ & 1.4199 \\
12 & $\left\{a_{4}, a_{5}, a_{6}\right\}$ & $\{0.704+0.564+0.4112\}$ & 1.6792 \\
\hline
\end{tabular}

TABLe 7: Spanning paths and sum of the score functions of their entries when $q=5$.

\begin{tabular}{lccc}
\hline Serial no. & $T_{i}=\left\{a_{i}\right\}$ & $\sum\left\{\mathcal{S}_{2}\left(a_{i}\right)\right\}$ & $\overline{T_{i}}$ \\
\hline 1 & $\left\{a_{1}, a_{2}, a_{3}\right\}$ & $\{0.4960+0.5684+0.4584\}$ & 1.5228 \\
2 & $\left\{a_{1}, a_{2}, a_{4}\right\}$ & $\{0.4960+0.5684+0.6636\}$ & 1.728 \\
3 & $\left\{a_{1}, a_{3}, a_{4}\right\}$ & $\{0.4960+0.4584+0.6636\}$ & 1.6174 \\
4 & $\left\{a_{1}, a_{3}, a_{5}\right\}$ & $\{0.4960+0.4584+0.5387\}$ & 1.4931 \\
5 & $\left\{a_{1}, a_{3}, a_{6}\right\}$ & $\{0.4960+0.4584+0.4315\}$ & 1.3859 \\
6 & $\left\{a_{1}, a_{5}, a_{6}\right\}$ & $\{0.4960+0.5387+0.4315\}$ & 1.4662 \\
7 & $\left\{a_{2}, a_{3}, a_{4}\right\}$ & $\{0.5684+0.4584+0.6636\}$ & 1.6904 \\
8 & $\left\{a_{2}, a_{4}, a_{5}\right\}$ & $\{0.5684+0.6636+0.5387\}$ & 1.7707 \\
9 & $\left\{a_{2}, a_{4}, a_{6}\right\}$ & $\{0.5684+0.6636+0.4315\}$ & 1.6635 \\
10 & $\left\{a_{2}, a_{5}, a_{6}\right\}$ & $\{0.5684+0.5387+0.4315\}$ & 1.5386 \\
11 & $\left\{a_{3}, a_{5}, a_{6}\right\}$ & $\{0.4584+0.5387+0.4315\}$ & 1.4286 \\
12 & $\left\{a_{4}, a_{5}, a_{6}\right\}$ & $\{0.6636+0.5387+0.4315\}$ & 1.6338 \\
\hline
\end{tabular}

in [43] (Section 4) to prove the efficiency of $q$-ROFMs and our proposed Algorithm 1, . It can be seen easily that the exiting method used to solve an application discussed in [43] (see Section 4) fails to solve the developed application in this study (see Section 4). Thus, for comparison, we use the dataset from Application (Section 4, Figure 2) [43]. Then, for $q=2,3,4$ and $q=5$, we compute the score functions (see Table 3 ). From Tables $4-7$, it can be easily seen that for any $q$, the spanning path $\left\{a_{1}, a_{3}, a_{6}\right\}$ attains the minimum value. Thus, our proposed technique is more flexible and generalized as it allows the decision makers to choose different values of $q$ according to the given fuzzy information. Moreover, we have used directed graphs, spanning trees, and maximal independent sets to propose a particular algorithm (Algorithm 1, ) that can be helpful in solving the human trafficking-related problems.

\section{Conclusion}

The study was carried out to enhance the real-life efficiency of some important models by curbing the issues of imprecise and vague information. Since the fuzzy sets are known to have the capacity to provide different models and tools for handling such information, $q$-ROFS is more suitable than IFS and PFS as it increases the space containing acceptable orthopair by increasing the value of parameter $q$. In this study, we have proposed vector spaces based on $q$-ROFSs and subsequently named them as $q$-ROFVSs. We have also discussed $q$-ROFVSs with an illustrative numerical example and developed some relevant results like basis and dimension. The $q$-ROF linearly independent vectors are also discussed. Furthermore, we have introduced $q$-ROFMs with their characteristics. We have extended some of the results based on IFM and PFM to $q$-ROFM. We have also discussed the notions of circuits, basis, and rank function for $q$-ROFMs. Finally, we have concluded the proposed work with a real-life application of decision making regarding human trafficking between different countries. For that, we used a directed graph with $q$-rung orthopair fuzzy information and combined it with the concept of maximal independent sets of edges of the graph to find the heaviest path. To enhance the capability of the $q$-ROFMs, we have provided a comparative analysis with an existing model. We are of the view that the given study would help the concerned countries in deciding the action response in a suitable path for the reduction of human flow. The major limitation of the proposed model is that it fails when the objects are evaluated concerning multiple parameters from more than one expert. In other words, there is no parametrization tool present in the initiated approach. Moreover, we are also interested to broaden our work to (a) $q$-rung orthopair fuzzy soft matroids, (b) fuzzy $N$-soft matroids, and (c) spherical fuzzy $\mathrm{N}$-soft matroids. This will illustrate more exclusive results based on the given fuzzy matroids and will be helpful in figuring out more real-life problems.

\section{Data Availability}

The data that support the findings of this study are available on request from the corresponding author.

\section{Conflicts of Interest}

The authors declare that they have no conflicts of interest.

\section{Acknowledgments}

This study was funded by the Deanship of Scientific Research (DSR) at King Abdulaziz University, Jeddah. The authors, therefore, acknowledge with thanks DSR for technical and financial support.

\section{References}

[1] H. Whitney, "On the abstract properties of linear dependence," American Journal of Mathematics, vol. 57, no. 3, pp. 509-533, 1935.

[2] L. A. Zadeh, "Fuzzy sets," Information and Control, vol. 8, no. 3, pp. 338-353, 1965.

[3] K. T. Atanassov, "Intuitionistic fuzzy sets," Fuzzy Sets and Systems, vol. 20, no. 1, pp. 87-96, 1986.

[4] F. Feng, H. Fujita, M. I. Ali, R. R. Yager, and X. Liu, "Another view on generalized intuitionistic fuzzy soft sets and related multiattribute decision making methods," IEEE Transactions on Fuzzy Systems, vol. 27, no. 3, pp. 474-488, 2018. 
[5] R. R. Yager, "Pythagorean fuzzy subsets," in Proceedings of the 2013 Joint IFSA World Congress and NAFIPS Annual Meeting, pp. 57-61, Edmonton, AB, Canada, June 2013.

[6] R. R. Yager, "Pythagorean membership grades in multicriteria decision making," IEEE Transactions on Fuzzy Systems, vol. 22, no. 4, pp. 958-965, 2014.

[7] K. T. Atanassov and P. Vassilev, "On the intuitionistic fuzzy sets of n-th type," Advances in Data Analysis with Computational Intelligence Methods, vol. 13, pp. 265-274, 2017.

[8] G. Bakioglu and A. O. Atahan, "AHP integrated TOPSIS and VIKOR methods with Pythagorean fuzzy sets to prioritize risks in self-driving vehicles," Applied Soft Computing, vol. 99, no. 2021, Article ID 106948, 2021.

[9] X. Liu, H. Kim, F. Feng, and J. C. R. Alcantud, "Centroid transformations of intuitionistic fuzzy values based on aggregation operators," Mathematics, vol. 6, Article ID 215, 2018.

[10] D. Pamucar and F. Ecer, "Prioritizing the weights of the evaluation criteria under fuzziness: the fuzzy full consistency method-fucom-f," Facta Universitatis-Series: Mechanical Engineering, vol. 18, no. 3, pp. 419-437, 2020.

[11] X. Peng and Y. Yang, "Some results for Pythagorean fuzzy sets," International Journal of Intelligent Systems, vol. 30, no. 11, pp. 1133-1160, 2015.

[12] R. Srinivasan and S. S. Begum, "Some properties of intuitionistic fuzzy sets of third type," International Journal of Humanities and Social Science, vol. 1, pp. 53-58, 2015.

[13] R. Srinivasan and K. Venkatesan, "Properties of intuitionistic fuzzy sets of fourth type," International Journal of Current Science and Research, vol. 3, pp. 21-25, 2017.

[14] R. R. Yager and A. M. Abbasov, "Pythagorean membership grades, complex numbers, and decision making," International Journal of Intelligent Systems, vol. 28, no. 5, pp. 436452, 2013.

[15] R. R. Yager, "Generalized orthopair fuzzy sets," IEEE Transactions on Fuzzy Systems, vol. 25, no. 5, pp. 1222-1230, 2017.

[16] H. Garg, "CN-q-Rofs: Connection number-based q-rung orthopair fuzzy set and their application to decision-making process," International Journal of Intelligent Systems, vol. 36, pp. 3106-3143, 2021.

[17] H. Garg, "New exponential operation laws and operators for interval-valued $q$-rung orthopair fuzzy sets in group decision making process," Neural Computing \& Applications, vol. 33, no. 1, 2021.

[18] G. Ali, H. Alolaiyan, D. Pamucar, M. Asif, and N. Lateef, "A novel MADM framework under $q$-Rung orthopair fuzzy bipolar soft sets," Mathematics, vol. 9, Article ID 2163, 2021.

[19] H. Garg, "A new possibility degree measure for intervalvalued $q$-rung orthopair fuzzy sets in decision-making," International Journal of Intelligent Systems, vol. 36, pp. 526-557, 2021.

[20] R. Verma, "Multiple attribute group decision-making based on order- $\alpha$ divergence and entropy measures under $q$-rung orthopair fuzzy environment," International Journal of Intelligent Systems, vol. 35, no. 4, pp. 718-750, 2020.

[21] S. Zeng, Y. Hu, and X. Xie, " $q$-rung orthopair fuzzy weighted induced logarithmic distance measures and their application in multiple attribute decision making," Engineering Applications of Artificial Intelligence, vol. 100, pp. 0952-1976, Article ID 104167, 2021.

[22] A. Kaufmann, Introduction a la Thiorie des Sous-Ensemble Flous, Masson, Paris, France, 1977.

[23] A. Rosenfeld, L. A. Zadeh, K. S. Fu, K. Tanaka, and M. Shimura, "FUZZY GRAPHS $\dagger \dagger$ The support of the Office of
Computing Activities, National Science Foundation, under Grant GJ-32258X, is gratefully acknowledged, as is the help of Shelly Rowe in preparing this paper," in Fuzzy Sets and Their Applications to Cognitive and Decision Processes, pp. 77-95, Academic Press, New York, NY, USA, 1975.

[24] M. Akram and S. Naz, "Energy of Pythagorean fuzzy graphs with applications," Mathematics, vol. 6, Article ID 560, 2018.

[25] M. Sitara, M. Akram, and M. Riaz, "Decision-making analysis based on q-rung picture fuzzy graph structures," International Journal of Applied Mathematics and Computer Science, vol. 67, 2021.

[26] M. Akram and W. A. Dudek, "Intuitionistic fuzzy hypergraphs with applications," Information Sciences, vol. 218, pp. 182-193, 2013.

[27] M. Akram and M. Sarwar, "Transversals of m-polar fuzzy hypergraphs with applications," Journal of Intelligent and Fuzzy Systems, vol. 33, no. 1, pp. 351-364, 2017.

[28] M. Akram and A. Luqman, "Bipolar neutrosophic hypergraphs with applications," Journal of Intelligent and Fuzzy Systems, vol. 33, no. 3, pp. 1699-1713, 2017.

[29] H. Lee-Kwang and K.-M. Lee, "Fuzzy hypergraph and fuzzy partition," IEEE Transactions on Systems, Man, and Cybernetics, vol. 25, no. 1, pp. 196-201, 1995.

[30] A. Luqman, M. Akram, and A. N. Al-Kenani, " $q$-Rung orthopair fuzzy hypergraphs with applications," Mathematics, vol. 7, Article ID 260, 2019.

[31] R. Parvathi, S. Thilagavathi, and M. G. Karunambigai, "Intuitionistic fuzzy hypergraphs," Cybernetics and Information Technologies, vol. 9, pp. 46-53, 2009.

[32] R. Goetschel and W. Voxman, "Fuzzy matroids," Fuzzy Sets and Systems, vol. 27, no. 3, pp. 291-302, 1988.

[33] R. Goetschel and W. Voxman, "Bases of fuzzy matroids," Fuzzy Sets and Systems, vol. 31, no. 2, pp. 253-261, 1989.

[34] R. Goetschel and W. Voxman, "Fuzzy circuits," Fuzzy Sets and Systems, vol. 32, no. 1, pp. 35-43, 1989.

[35] R. Goetschel and W. Voxman, "Fuzzy rank functions," Fuzzy Sets and Systems, vol. 42, no. 2, pp. 245-258, 1991.

[36] R. Goetschel and W. Voxman, "Fuzzy matroid structures," Fuzzy Sets and Systems, vol. 41, no. 3, pp. 343-357, 1991

[37] Y. C. Hsueh, "On fuzzification of matroids," Fuzzy Sets and Systems, vol. 53, pp. 317-327, 1993.

[38] S.-G. Li, X. Xin, and Y.-L. Li, "Closure axioms for a class of fuzzy matroids and co-towers of matroids," Fuzzy Sets and Systems, vol. 158, no. 11, pp. 1246-1257, 2007.

[39] X. Li and H. Yi, "Axioms for fuzzy bases of Hsueh fuzzy matroids," Journal of Intelligent and Fuzzy Systems, vol. 29, no. 5, pp. 1995-2001, 2015.

[40] X. Li and H. Yi, "Intuitionistic fuzzy matroids," Journal of Intelligent and Fuzzy Systems, vol. 33, no. 6, pp. 3653-3663, 2017.

[41] Y. Li, L. Li, J. Li, D. Qiu, and H. Duan, "Bases of G-V intuitionistic fuzzy matroids," Mathematics, vol. 8, Article ID 1392, 2020.

[42] F.-G. Shi, "A new approach to the fuzzification of matroids," Fuzzy Sets and Systems, vol. 160, no. 5, pp. 696-705, 2009.

[43] M. Asif, M. Akram, and G. Ali, "Pythagorean fuzzy matroids with application," Symmetry, vol. 12, Article ID 423, 2020.

[44] A. K. Katsaras and D. B. Liu, "Fuzzy vector spaces and fuzzy topological vector spaces," Journal of Mathematical Analysis and Applications, vol. 58, no. 1, pp. 135-146, 1977.

[45] J. N. Mordeson and S. Mathew, "Non-deterministic flow in fuzzy networks and its application in identification of human trafficking chains," New Mathematics and Natural Computation, vol. 3, pp. 1-13, 2017. 